\title{
Study and analysis of motion artifacts for ambulatory electroencephalography
}

\author{
Asma Islam ${ }^{1}$, Eshrat Jahan Esha1, Sheikh Farhana Binte Ahmed ${ }^{2}$, Md. Kafiul Islam ${ }^{1}$ \\ ${ }^{1}$ Department of Electrical and Electronic Engineering, Independent University, Bangladesh, Dhaka, Bangladesh \\ ${ }^{2}$ Department of Electrical and Electronic Engineering, Islamic University of Technology, Gazipur, Bangladesh
}

\begin{abstract}
Article Info
Article history:

Received Jun 9, 2021

Revised Sep 9, 2021

Accepted Oct 4, 2021

\section{Keywords:}

Electroencephalogram

Electroencephalography

Motion artifact

Movement artifact

Neural signal

Scalp EEG

ABSTRACT

Motion artifacts contribute complexity in acquiring clean electroencephalography (EEG) data. It is one of the major challenges for ambulatory EEG. The performance of mobile health monitoring, neurological disorders diagnosis and surgeries can be significantly improved by reducing the motion artifacts. Although different papers have proposed various novel approaches for removing motion artifacts, the datasets used to validate those algorithms are questionable. In this paper, a unique EEG dataset was presented where ten different activities were performed. No such previous EEG recordings using EMOTIV EEG headset are available in research history that explicitly mentioned and considered a number of daily activities that induced motion artifacts in EEG recordings. Quantitative study shows that in comparison to correlation coefficient, the coherence analysis depicted a better similarity measure between motion artifacts and motion sensor data. Motion artifacts were characterized with very low frequency which overlapped with the Delta rhythm of the EEG. Also, a general wavelet transform based approach was presented to remove motion artifacts. Further experiment and analysis with more similarity metrics and longer recording duration for each activity is required to finalize the characteristics of motion artifacts and henceforth reliably identify and subsequently remove the motion artifacts in the contaminated EEG recordings.
\end{abstract}

This is an open access article under the CC BY-SA license.

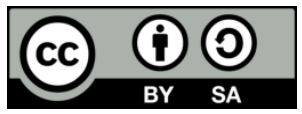

\section{Corresponding Author:}

Md. Kafiul Islam

Department of Electrical and Electronic Engineering, School of Engineering, Technology and Sciences Independent University, Bangladesh

Academic Block, Level 4 and 5, Plot-16, Block-B, Bashundhara, Dhaka-1229, Bangladesh

Email: kafiul_islam@iub.edu.bd

\section{INTRODUCTION}

According to the estimation of World Health Organization (WHO), hundreds of millions of people are suffering from neurological disorders globally. Approximately more than 47 million people have dementia and around 50 million are affected by epilepsy. Around one in every 100 persons will encounter a seizure at some point in their lifetime [1]. Most of these neurological disorders require long term electroencephalography (EEG) monitoring for diagnosis and treatment.

Modern neuroscience demands such a way to record brain dynamics where patients are allowed to perform their natural actions and everyday activities in their regular way. Traditional non-invasive EEG recording techniques such as functional magnetic resonance imaging (fMRI), positron emission tomography (PET), magnetoencephalography (MEG), and single photon emission spectroscopy (SPET) require restricted whole-body movement and restrained head positioning [2]. When it comes to recording continuous brain 
activities, a comfortable monitoring system is incumbent where a patient is not required to be in the clinic or any constrained body position. Ambulatory EEG fulfills that requirement. Surface EEG is the best technological option that entitles one to record brain activities during mechanical movement [3], [4]. Ambulatory EEG monitoring can contribute to smart home environments, hospitals and healthcare centers with the help of the integration of the internet of things (IoT) which will increase interoperability, expansibility, and intelligence [5]. Using watermarking technique, protected transmission of EEG data is possible [6], [7].

However, the vital requirement of ambulatory EEG is to ensure high quality of EEG signals in a real-time environment. Fulfilling this requirement is being considered as one of the major hurdles in ambulatory EEG. Brain signals get easily contaminated by various artifacts and noises occurring by body movements. Motion artifacts relatively have a greater impact on ambulatory EEG as it shares the same frequency spectra with EEG signal (up to $50 \mathrm{~Hz}$ ) but has a larger amplitude than the brain signals [8]. It is also highly associated with the movements of the subjects and experimental devices. Motion artifacts cause significant deviation of the EEG data and manipulate it by changing the original shape of the signal. This leads to misinterpretation of the EEG data and thus wrong diagnosis of diseases, and false alarms. During motion, voltage fluctuations occur due to the movements of electrodes from their standard position or cable sway. These motion artifacts can occur from different sources like ground reaction forces, cyclic motion, and head movements. Thus, motion artifacts heavily corrupt the raw EEG data if the recording is carried out while doing daily activities which can range from high to low power spectral density [9]. By removing the motion artifacts from recorded mobile EEG data, it will enhance the performance of error reduction, classification [10], diagnosis and brain computer interfaces (BCI) applications.

In existing papers on motion artifact removal, the trickiest part is the dataset. Lack of standardized, validated and highly integrated datasets of biomedical signals is still a crucial need for researchers [11]. Different techniques for removing artifacts from the EEG signals have been done already. Yet, very little research work has been done on removing motion artifacts from ambulatory EEG. Most of the papers either concentrate only on detecting motion artifacts from EEG or reducing artifacts only for a particular regular activity that prompted motion artifact. Different multi-resolution techniques were demonstrated in papers such as singular spectrum analysis (SSA) [12], discrete wavelet transform (DWT) [13], empirical mode decomposition (EMD) [14], ensemble empirical mode decomposition with canonical [15], canonical correlation analysis (CCA) [16], total variation (TV) and weighted total variation (WTV) [17]. Besides multiresolution techniques [18] they have adopted impedance measurement of dry electrodes approach to eliminate the motion artifacts. But the effectiveness of proposed techniques is being questioned as the information about experimental setup, tasks performed by the subjects, quality of the instrumentations of most of the published datasets remain indeterminate. These regardless restrains to do a comparative study and behavior analysis of algorithms on the available techniques as they have used different datasets, softwares and parameters to check the algorithm behavior. The dataset by Sweeney et al. is most commonly used by the researchers to evaluate their proposed methods in [13]-[15], [17], [19] which is available in physionet online platform. In that dataset, one of the two channels was used to record motion artifact contaminated EEG signal and the other channel was used to record motion artifact data. 23 trials of EEG signals were recorded from six healthy subjects. Subjects did not perform any ambulatory activities. They were instructed to keep their head at a stationary position and remain their eyes closed throughout the experiment which limited the EOG artifacts and head movement artifacts [20].

Dataset by Shoeb [21] has been used to check the efficacy of removing motion artifacts in [16], although the epileptic seizure recordings from real patients do not contain any marker or defined presence of motion artifacts. Inducing artifacts by pulling the connecting lead was considered a motion artifact [12], [19], [20]. These datasets do not reciprocate and ensure the natural characteristics of "real" motion artifacts for ambulatory movements. The researcher in [22] has opted synthetic sinusoidal signal to represent the EEG signal. However, some papers used the private datasets that included either walking [8], [23]-[25] or head movements [18], [26]-[28] for the quantitative validation of their performance to reduce motion artifacts. Only a few authors have created the datasets with the versatile activities that induced motion artifact contaminated raw EEG signal [29]. These performed activities do not involve both lower and upper body movements. The diversity in activities presented in these papers and the absence of a standardized dataset that contains defined motion artifacts only permit the self-evaluation of any algorithm while leaving no room for the peer review to evaluate and compare the performance. The obliviousness of these datasets used in these papers restrains the scope of doing a comparative study on the available techniques since they have used different datasets to assess the performance parameters. Thus, to overcome this problem, we have generated novel datasets that confirm the presence of motion artifacts with the necessary markers in the EEG signal using the EMOTIV device. The dataset will be able to serve the researchers to measure standard performance parameters among the existing methods for reducing motion artifacts. In addition, a general algorithm has been proposed to reduce motion artifacts based on our quantitative analysis. The datasets 
ensure that our proposed algorithm removes motion artifacts using ' $\mathrm{db} 2$ ' wavelet decomposition on the A4 level.

\section{MATERIALS AND METHODS}

We have used EMOTIV EPOC+, a multichannel neuroelectric signal recording device with built-in accelerometer, gyroscope, and magnetometer sensors. To maintain the conductance and stability, skin saline solution is used on electrodes for its satisfactory properties within the lower frequency spectrum [30]. This wireless device is able to continuously record EEG data at a sampling frequency of $2048 \mathrm{~Hz}$.

Six healthy male volunteers have participated who age between 23 to 25 years. Participants were confirmed with no medical complications or neurological deficits. Before performing the activities, participants were being informed about the experiment and its privacy. They had signed a consent paper. It took four days to record the 6 sets of trials. Each trial was 40 minutes long which totals 240 minutes. The recording was done in a normal environment but the activities were highly monitored in order to record the artifacts that were created for the particular ambulatory activity. Participants were instructed to perform the activities twice with electrodes and without electrodes attached to the headset arms. The activities were divided into sets (1) relax-considered as reference or motion artifact free signal (2) activity-that produces motion artifact. Delay session was subject-dependent where the subject was allowed to move of their choice. Participants had freedom with their eye movements in first two recordings but for more precision the activities were then divided into two sub-sections opened and closed eyes. For proper interpretation and uniformity of the datasets being used for the analysis, the first two datasets were not mentioned in the results section. Figure 1 shows the basic structure of the experiment. After performing the activities with electrodes attached to the headset, we removed the electrodes from the headset to perform the same set of activities again. The daily activities were selected which induced motion artifacts. Participants were restricted from any movements other than the particular activity so that only the artifact for a particular activity gets recorded and time was marked for the respective activities. The uniqueness of this particular dataset is that it combines head, lower body and cyclical movements. The duration between each sub-section was 15 seconds and for each activity was 30 seconds. Ten types of activities were performed by each subject such as head tilting, head shaking, head nodding, standing up and sitting down, walking, bending, leg trembling, walking stairs, talking and relax as shown in Figures 2(a)-(i). Figure 2 shows a visual representation of these activities respectively. Multiple relaxation sessions were performed by each participant to acquire better reference data. The activities took seventeen minutes each excluding delays. Participants were forbidden to speak during activities.

We proceeded with the approach of reducing motion artifacts with two hypotheses based on the analysis. The first hypothesis is that motion artifacts tend to have higher amplitude than background EEG. Based on this hypothesis, detection of motion artifacts can be performed while applying thresholding in wavelet based denoising. Signal components which have higher values than the particular threshold is passed for further detection and removal of the artifacts, but if it is lower than the threshold then it is preserved assuming background EEG activity. The second hypothesis is that the motion artifact is most likely to be in delta rhythm ranging up to $3 \mathrm{~Hz}$. So, evaluating performance on A4 (0-4 Hz) decomposition level for the detection and reduction of motion artifacts from the signal was a prudent choice. Keeping all of these into consideration we have proposed an algorithm that is able to identify and remove the motion artifacts from the contaminated EEG data. Figure 3 demonstrates the flow chart of the proposed algorithm.

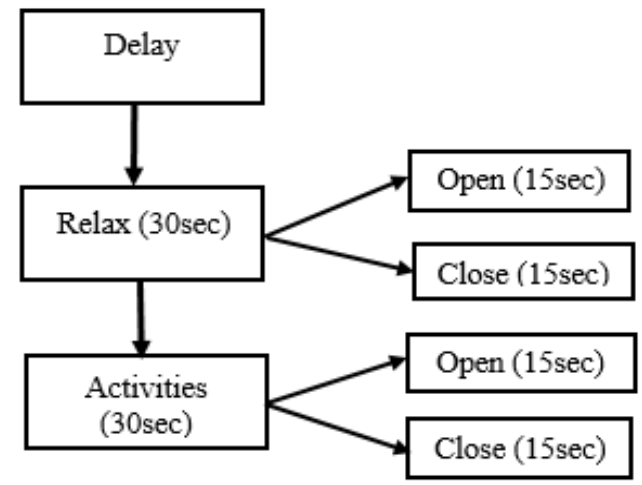

Figure 1. Basic structure of experiment for recording EEG signals to acquire different motion activities 


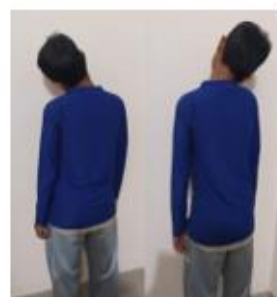

(a)

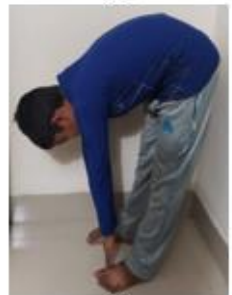

(f)

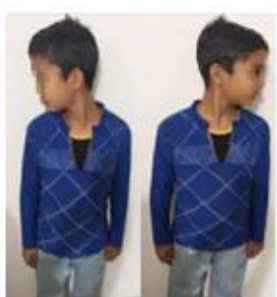

(b)

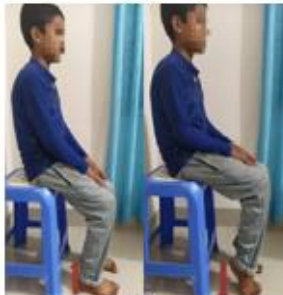

(g)

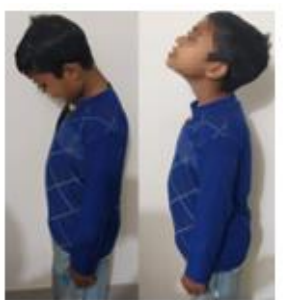

(c)

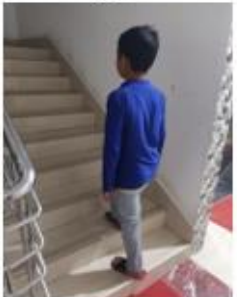

(h)

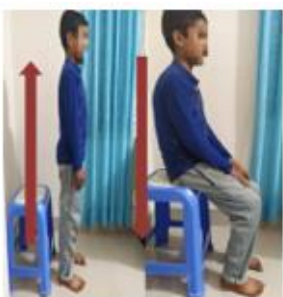

(d)

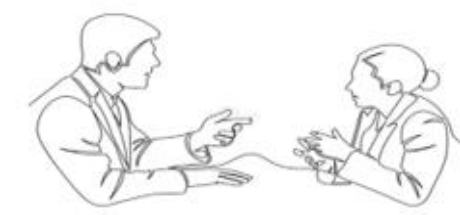

(i)

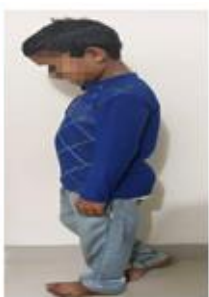

(e)

Figure 2. Mostly performed daily activities which contaminate EEG recordings with motion artifacts;

(a) head tilting, (b) head shaking, (c) head nodding, (d) stand up and sit down, (e) walking,

(f) bending, (g) leg trembling, (h) walking on stairs, and (i) talking

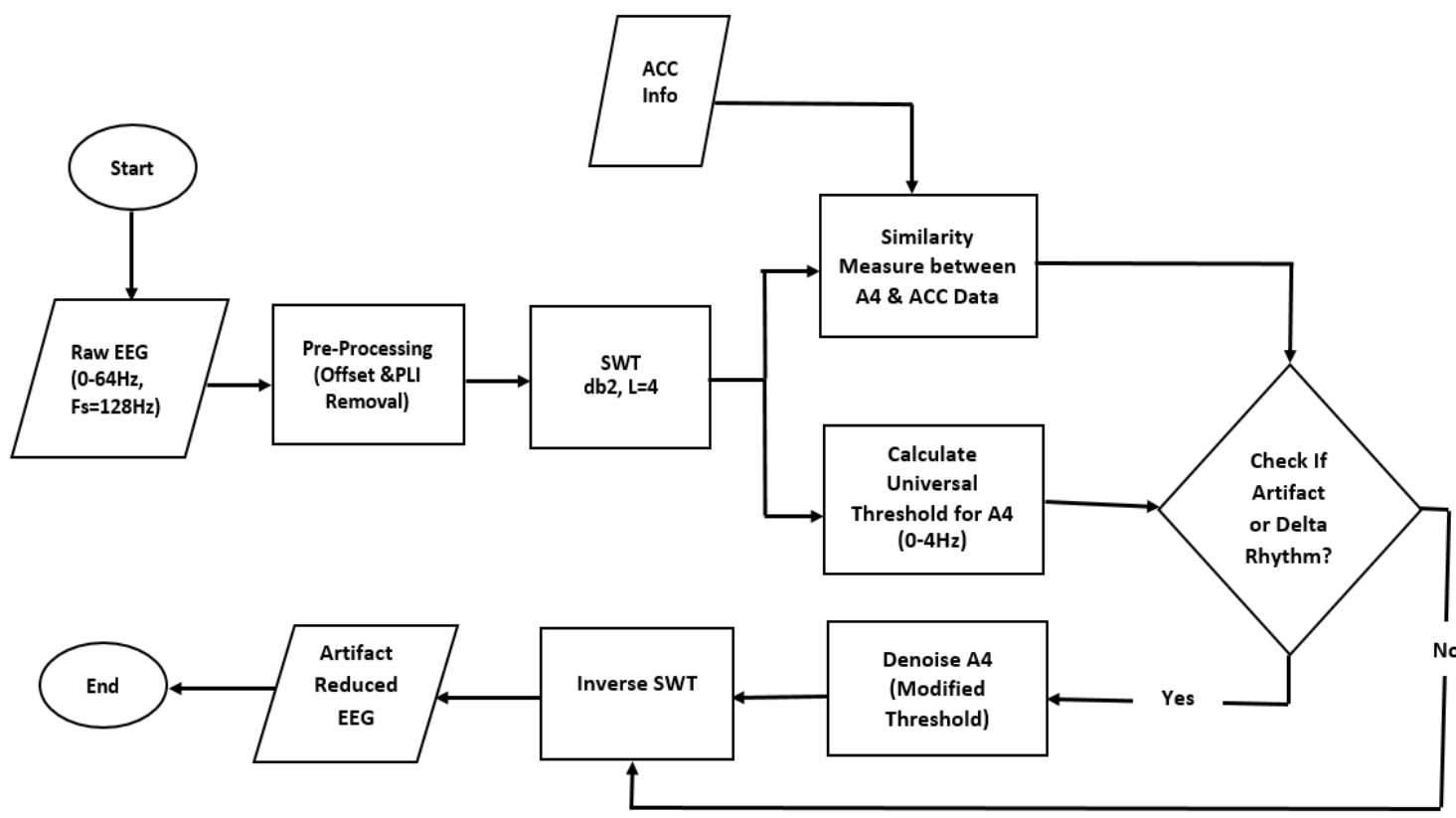

Figure 3. Flow chart of the proposed algorithm

The motion artifact contaminated signals were segmented into 120 seconds epochs. Raw EEG data was contaminated with different noises, artifacts, dc offset and other factors. To remove powerline interference a $50 \mathrm{~Hz}$ notch filter is used. To remove the dc offset, the mean of the raw signals is subtracted from the EEG raw signal. We have used the "db2" wavelet on level 4 for stationary wavelet transform (SWT) decomposition. SWT is considered to be one of the most powerful methods to reduce artifacts while preserving the neural information of EEG signals. We have used different average coefficient ratios to decide the threshold level and then preferred it to be 2.5 , at which the algorithm performed most efficiently in terms of motion artifact removal. The coherence coefficient is calculated between accelerometer data and EEG signal decomposed at A4 level to measure the similarities. The next step is to check if the EEG signal at A4 level is an artifact or delta rhythm by comparing it with accelerometer data. If the answer is delta, the signal decomposed at A4 level was reconstructed by performing inverse SWT and if it is an artifact, the signal was denoised with modified thresholding before reconstructing and thus the artifact is reduced. 


\section{RESULTS AND DISCUSSION}

We have analyzed and evaluated the recorded dataset consisting of 4 sets of trials with and without electrodes alongside defined opened and closed eyes. We have done this using correlation coefficient, coherence, rhythm and wavelet transform for the particular activities to characterize motion artifacts.

\subsection{Results}

Table 1 demonstrates the correlation coefficient between EEG signal (with electrodes) and accelerometer data. The activities during open eyes condition are indicated by the letter " $O$ " and the activities during closed eyes condition are indicated by the letter " $C$ ". The resultant value did not exhibit any significant relation between the neural and motion sensor data. The signals are negatively correlated with each other as the phase shift in the time domain is encountered.

Table 1. Correlation coefficient analysis between EEG data with electrode vs. ACC data for eyes open and eyes closed respectively

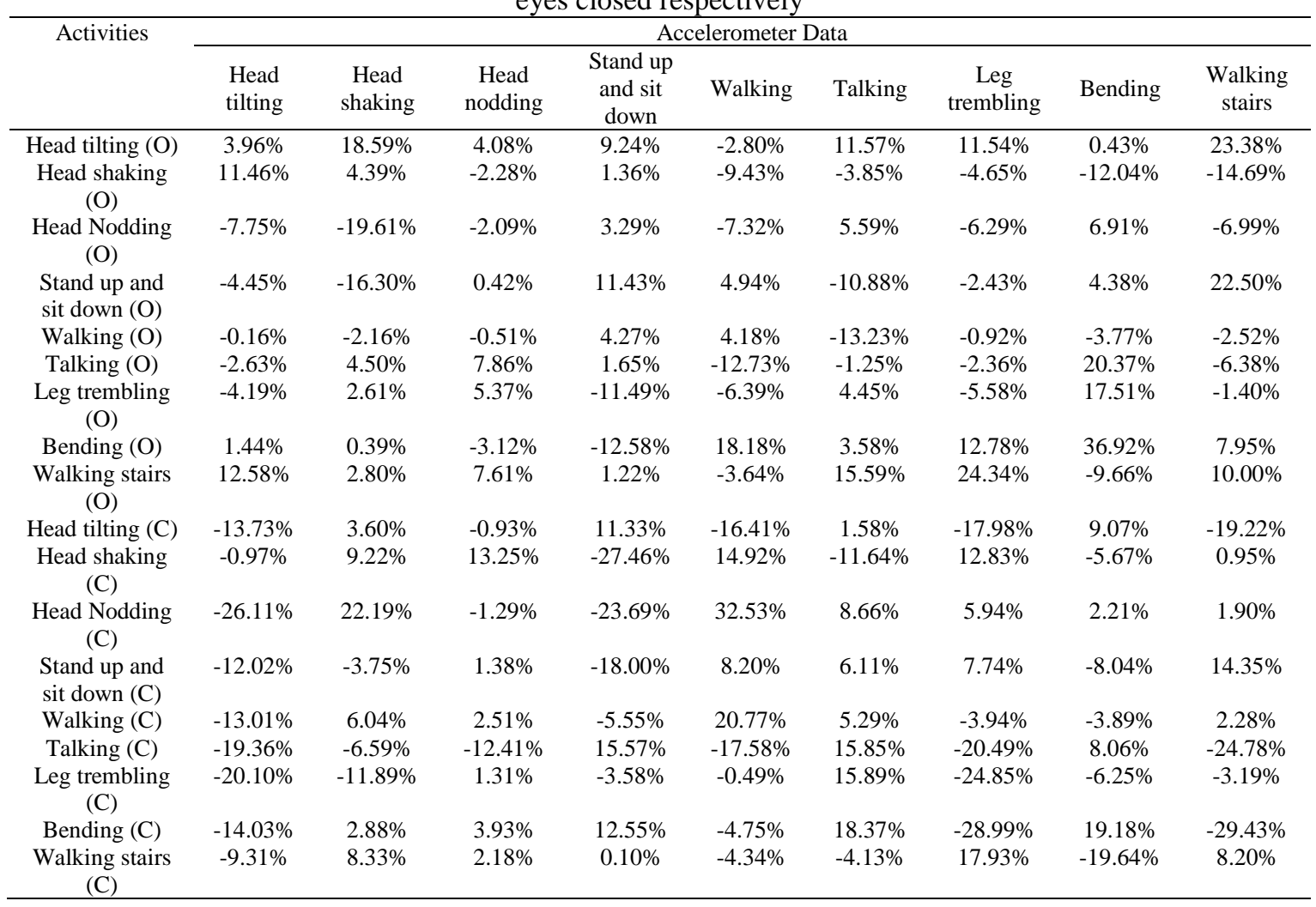

Table 2 illustrates the average coherence coefficient analysis for different activities with respect to the accelerometer data. The neural signals were recorded while the EEG electrodes were mounted on the scalp whereas the accelerometer sensor was attached and detached in separate sessions. The artifact in the contaminated EEG signal matches with the accelerometer information with a higher coherence coefficient value which confirms the presence of motion artifact of any particular activity. The highest coefficient that matches with the other activities are justified as some other activities have similarities in movement. The coherence coefficient is high for motion contaminated EEG data whereas for the clean EEG signal the coherence is low. Table 3 also shows the average coherence coefficient analysis for diverse movements along with relax session with respect to the accelerometer data. In this case, the measurements were taken without the EEG electrodes. Similar to Table 2, the accelerometer data was taken for both opened and closed condition. The results align with that of the session with electrodes.

Table 4 shows the relative band power for each EEG rhythm for the clean EEG signal and motion artifacts contaminated signals. This table allows a better comparison between activities and also with the clean data in terms of which waves are dominantly present in each of the activities. As we can see, the most dominant waveform is Delta rhythm. It contains most of the power in both clean and motion contaminated data. In contaminated EEG signals, the delta power gets increased by the presence of motion artifacts, 
ensuring its existence. From Table 4 the average delta rhythm is $96.09 \%$ in contaminated data and $90.38 \%$ is for clean data. Figure 4 demonstrates the zoomed view of the reference EEG signal and motion artifact contaminated signal. The bold line represents the contaminated data with the motion artifacts and the dotted line represents the clean EEG signal.

Table 2. Coherence coefficient analysis of EEG data with electrode VS ACC data

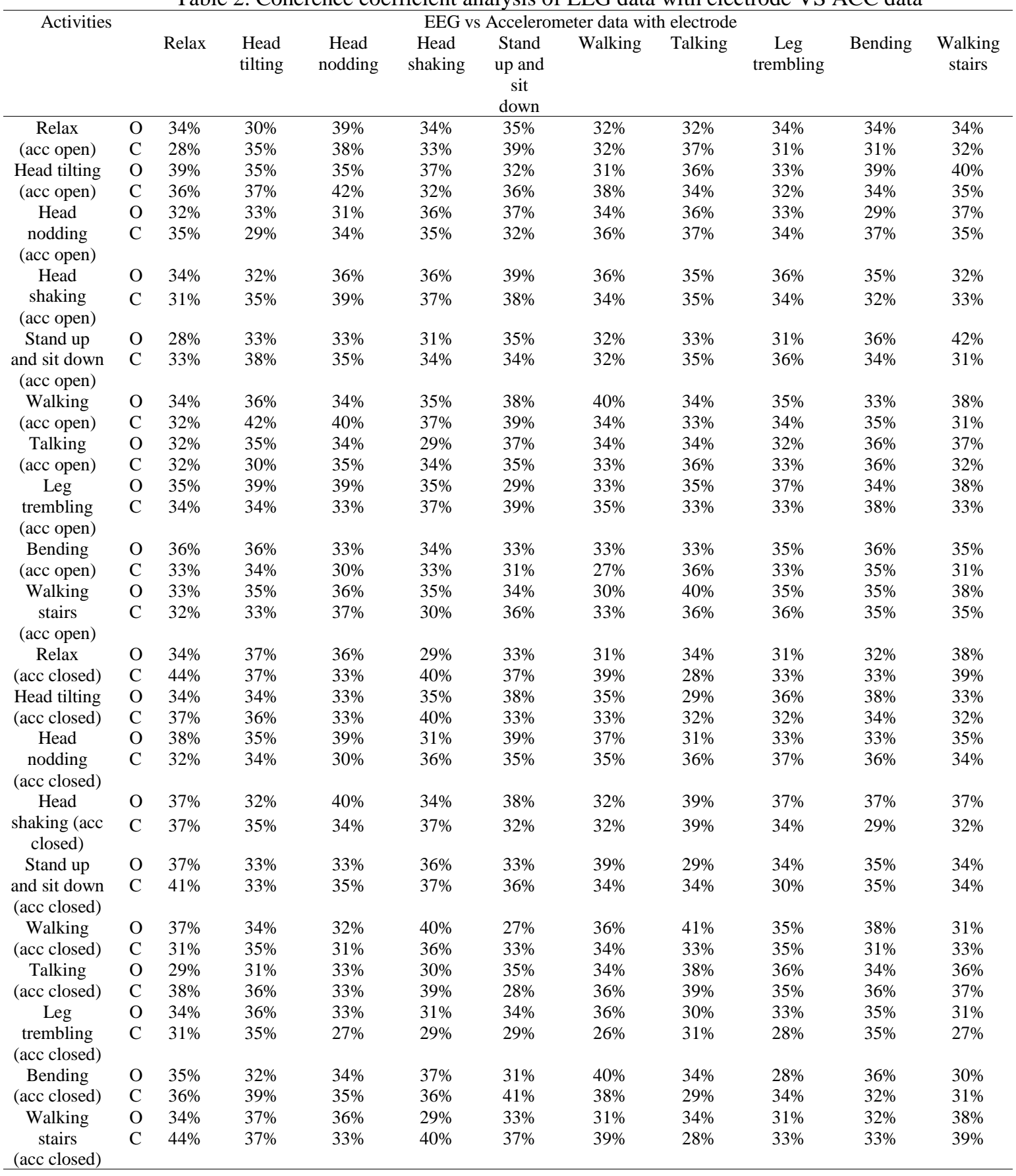

\subsection{Discussion}

The necessity of reliable datasets contaminated with motion artifacts and identifying them from ambulatory EEG was the prime target of our study. Online datasets of existing research work based on which the performance parameters are evaluated are questionable. The generation of artifacts that claimed motion artifacts was done by pulling the connecting lead which differed from the real-life ambulatory activities. 
Table 3. Coherence coefficient analysis of EEG data without electrode VS ACC data

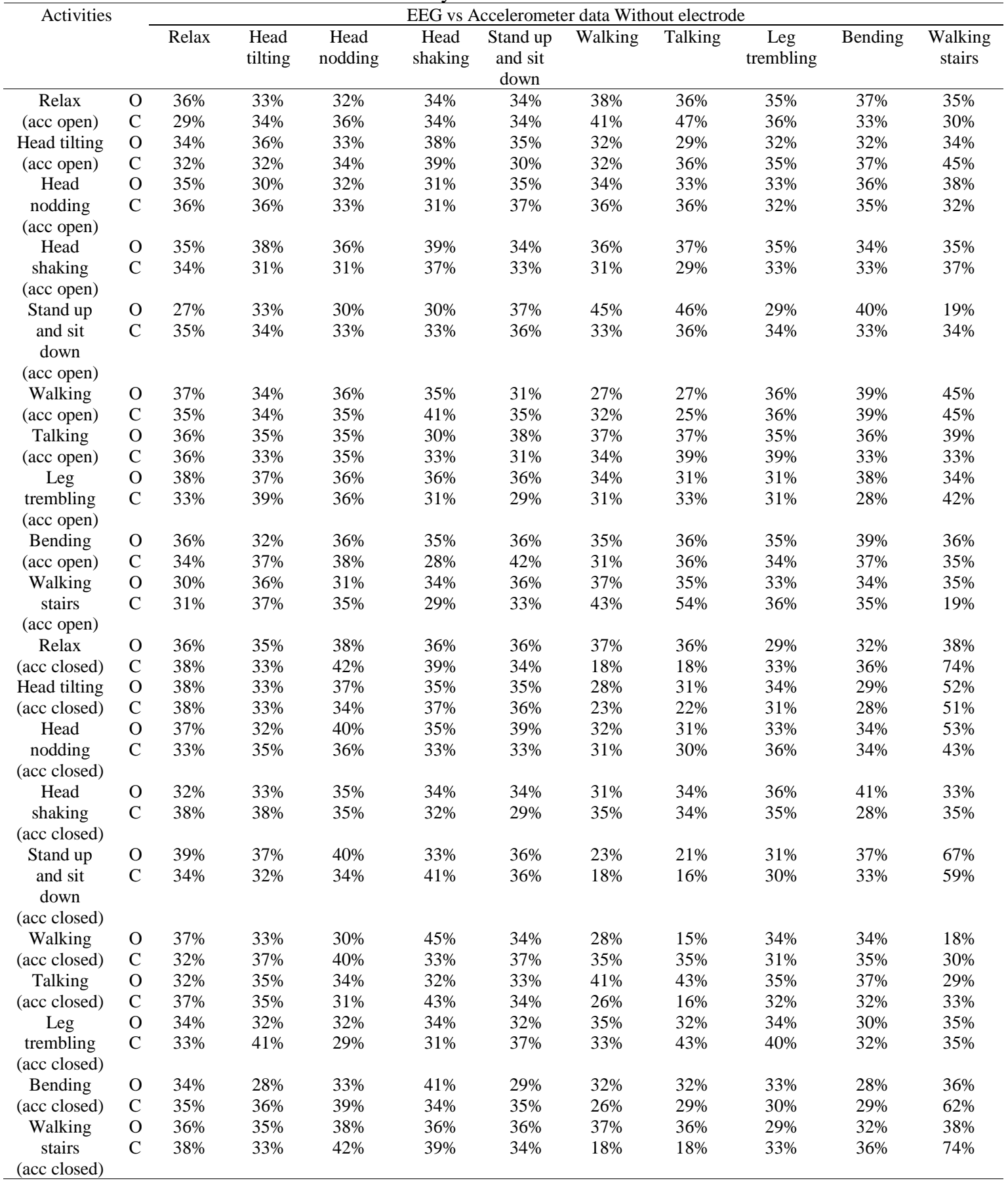

Table 4. Average power of EEG rhythms for clean EEG and motion artifacts contaminated EEG signals

\begin{tabular}{cccccccccccc}
\hline \multirow{2}{*}{$\begin{array}{c}\text { EEG } \\
\text { Rhythm }\end{array}$} & \multicolumn{10}{c}{ Activities } \\
\cline { 2 - 12 } & Relax & $\begin{array}{c}\text { Head } \\
\text { tilting }\end{array}$ & $\begin{array}{c}\text { Head } \\
\text { shaking }\end{array}$ & $\begin{array}{c}\text { Head } \\
\text { nodding }\end{array}$ & $\begin{array}{c}\text { Stand up and } \\
\text { sit down }\end{array}$ & Walking & Talking & $\begin{array}{c}\text { Leg } \\
\text { trembling }\end{array}$ & Bending & $\begin{array}{c}\text { Walking } \\
\text { stairs }\end{array}$ \\
\hline Delta & O & 90.37 & 97.33 & 96.67 & 97.57 & 98.41 & 97.67 & 98.09 & 95.68 & 96.68 & 96.95 \\
& $\mathrm{C}$ & 90.39 & 95.36 & 97.17 & 96.98 & 98.96 & 94.73 & 95.86 & 84.76 & 97.17 & 93.62 \\
Theta & $\mathrm{O}$ & 2.24 & 1.17 & 1.148 & 0.81 & 0.74 & 0.87 & 0.32 & 1.02 & 0.54 & 0.87 \\
& $\mathrm{C}$ & 0.95 & 1.51 & 0.81 & 0.63 & 0.32 & 1.38 & 0.55 & 1.97 & 0.24 & 3.43 \\
Alpha & $\mathrm{O}$ & 1.14 & 0.48 & 0.49 & 0.42 & 0.23 & 0.41 & 0.27 & 0.72 & 0.34 & 0.53 \\
& $\mathrm{C}$ & 0.8 & 1.34 & 0.83 & 1.08 & 0.16 & 0.55 & 0.78 & 6.36 & 0.29 & 1.84 \\
Beta & $\mathrm{O}$ & 1.45 & 0.62 & 0.81 & 0.56 & 0.28 & 0.46 & 0.34 & 0.86 & 0.43 & 0.79 \\
& $\mathrm{C}$ & 1.55 & 0.75 & 0.55 & 0.67 & 0.23 & 1.59 & 0.67 & 3.37 & 0.70 & 1.03 \\
Gamma & $\mathrm{O}$ & 5.03 & 0.59 & 0.67 & 1.09 & 0.43 & 0.69 & 1 & 1.83 & 2.59 & 0.63 \\
& $\mathrm{C}$ & 6.45 & 1.14 & 0.72 & 0.71 & 0.37 & 1.9 & 2.2 & 3.51 & 1.56 & 0.45 \\
\hline
\end{tabular}




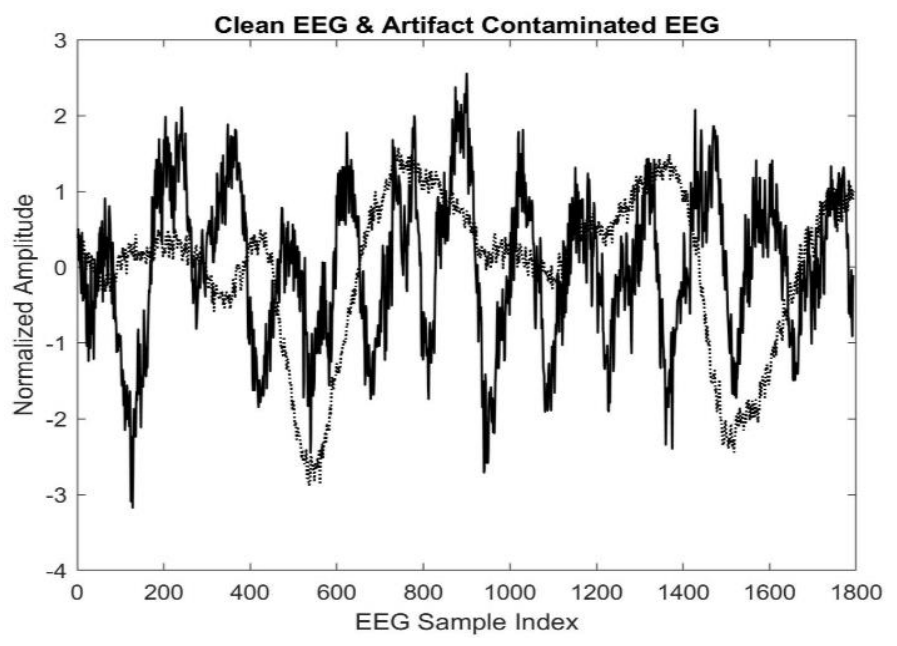

Figure 4. Clean EEG (dotted line) and contaminated EEG signal (bold line)

The datasets which were generated by authors in previous works have considered only particular types of movements. We proposed a solution by generating an extensive dataset that contains activities related to both upper and lower body movements. Ambulatory EEG signals get mostly altered by the motion artifacts. To reduce the motion artifacts from the neural signal, SWT is more proficient than the other techniques for removing an artifact from the neural signal. We have proposed an approach based on our analysis using a simple wavelet cleaning method that is insufficient to reduce all the motion artifacts from the EEG signal. The correlation coefficient analysis cannot be opted for the similarities with accelerometer data because of the phase shift in the signal as it compares signals in the time domain. We found almost no similarities between EEG data and accelerometer data where they are negatively related in some activities for the phase shift. However, from this analysis it can help to characterize the relationship between activities and how they are negatively co-related for intensive study. Coherence analysis compares the signal in the frequency domain. Therefore, the coherence coefficient approach was able to bridge relation between EEG and accelerometer data.

During the motion activities, the spectral power increases in the lower frequency band. We calculated the relative power of the percentage for each activity. Delta has the highest percentage in every activity and motion contaminated signals have higher delta rhythms than clean data. Leg trembling, bending head tilting got higher theta waves. Activities with lower body movements have higher alpha and beta waves. Using this information, detection and estimation of the level of mobility of artifacts can be done. Although the proposed model can be utilized to remove motion artifacts, it did not outperform the other techniques for various reasons. The results varied as different subjects had different speeds and patterns. We also found the conductance depends on the head size and hair which can contribute to the difference in electrode and accelerometer data. The sensors need to be placed on different parts of the body to record most accurate information of motion artifactual EEG data.

Since the ambulatory EEG demands a realistic ambience, in order to extract the accurate template, the activities needed to be performed in the restricted environment. Furthermore, the lack of resources contributed to not getting the expected result. The relax signal is also contaminated by some unavoidable circumstances. Even a little difference that is unable to be monitored can create voltage fluctuation in electrodes. Relative motion to the scalp in wireless electrode systems still contributes to artifacts. The recording sessions were too long due to the repetition of activities for both with and without electrodes which might be tiring for the subject causing some unavoidable motion. Some unwanted eye blinks and hand movement artifacts were induced in walking, bending and while walking stairs. The patterns can be more evident if the duration of each activity were at least one minute. Despite having these limitations, the proposed datasets ensure the presence of motion artifacts for ambulatory movements in the EEG signal.

\section{CONCLUSION}

The removal of motion artifacts is crucial from the EEG signal to improve the performance of the AEEG. To improve the efficacy of the existing or new method it is incumbent to have a reliable dataset that is recorded in a living environment. In this paper, we have introduced a new EEG dataset that is contaminated with real-time motion artifacts. Here, coherence serves as a better similarity measure between 
the motion artifacts contaminated EEG and the motion sensor data. This is supported by our results in Tables 1, 2 and 3. In Table 4 it was also observed that the motion artifacts coincide with the delta rhythm of EEG. The recordings are done in controlled supervision to identify each activity precisely. This dataset is particularly salient considering the lack of research work on removing motion artifact from the contaminated EEG signals. This real dataset is mainly contrived to assist researchers who are working on motion artifact removal techniques for AEEG so that they can fairly compare with the existing methods and improve new models. In future, we plan to extract templates for each activity and create them artificially by extracting and taking the envelope from neural signals for ambulatory activities. Then the artifact templates can be created into different amplitudes to do further study. Besides, we are also looking forward to upload the datasets on Physionet by end of 2021 in the form of CSV files after necessary labeling and formatting.

\section{REFERENCES}

[1] L. D. Iasemidis, "Epileptic seizure prediction and control," IEEE Transactions on Biomedical Engineering, vol. 50, no. 5, pp. 549-558, May 2003, doi: 10.1109/TBME.2003.810705.

[2] A. S. Oliveira, B. R. Schlink, W. D. Hairston, P. König, and D. P. Ferris, "Induction and separation of motion artifacts in EEG data using a mobile phantom head device," J. of Neural Engineering, vol. 13, no. 3, p. 36014, 2016, doi: 10.1088/17412560/13/3/036014.

[3] K. Gramann, D. P. Ferris, J. Gwin, and S. Makeig, "Imaging natural cognition in action," International Journal of Psychophysiology, vol. 91, no. 1, pp. 22-29, Jan. 2014, doi: 10.1016/j.ijpsycho.2013.09.003.

[4] P. M. R. Reis, F. Hebenstreit, F. Gabsteiger, V. von Tscharner, and M. Lochmann, "Methodological aspects of EEG and body dynamics measurements during motion," Frontiers in Human Neuroscience, vol. 8, Mar. 2014, doi: 10.3389/fnhum.2014.00156.

[5] A.-M. Rahmani et al., "Smart e-Health Gateway: Bringing intelligence to Internet-of-Things based ubiquitous healthcare systems," in 2015 12th Annual IEEE Consumer Communications and Networking Conference (CCNC), Jan. 2015, pp. 826-834, doi: 10.1109/CCNC.2015.7158084.

[6] A. Gupta, C. Chakraborty, and B. Gupta, "Monitoring of epileptical patients using cloud-enabled Health-IoT system," Traitement du Signal, vol. 36, no. 5, pp. 425-431, Nov. 2019, doi: 10.18280/ts.360507.

[7] A. K. Gupta, C. Chakraborty, and B. Gupta, "Secure transmission of EEG data using watermarking algorithm for the detection of Epileptical Seizures," Traitement du Signal, vol. 38, no. 2, pp. 473-479, Apr. 2021, doi: 10.18280/ts.380227.

[8] J. T. Gwin, K. Gramann, S. Makeig, and D. P. Ferris, "Removal of movement artifact from high-density EEG recorded during walking and running," Journal of Neurophysiology, vol. 103, no. 6, pp. 3526-3534, Jun. 2010, doi: 10.1152/jn.00105.2010.

[9] C. Wen, M.-F. Yeh, K.-C. Chang, and R.-G. Lee, "Real-time ECG telemonitoring system design with mobile phone platform," Measurement, vol. 41, no. 4, pp. 463-470, May 2008, doi: 10.1016/j.measurement.2006.12.006.

[10] M. Sameer, A. K. Gupta, C. Chakraborty, and B. Gupta, "Epileptical Seizure detection: Performance analysis of gamma band in EEG signal using short-time fourier transform," in 2019 22nd International Symposium on Wireless Personal Multimedia Communications (WPMC), Nov. 2019, pp. 1-6, doi: 10.1109/WPMC48795.2019.9096119.

[11] A. L. Goldberger et al., "PhysioBank, PhysioToolkit, and PhysioNet," Circulation, vol. 101, no. 23, Jun. 2000, doi: 10.1161/01.CIR.101.23.e215.

[12] A. K. Maddirala and R. A. Shaik, "Motion artifact removal from single channel electroencephalogram signals using singular spectrum analysis," Biomedical Signal Processing and Control, vol. 30, pp. 79-85, Sep. 2016, doi: 10.1016/j.bspc.2016.06.017.

[13] S. Shukla, V. Roy, and A. Prakash, "Wavelet based empirical approach to mitigate the effect of motion artifacts from EEG signal," in 2020 IEEE 9th International Conference on Communication Systems and Network Technologies (CSNT), Apr. 2020, pp. 323-326, doi: 10.1109/CSNT48778.2020.9115761.

[14] N. E. Huang et al., "The empirical mode decomposition and the Hilbert spectrum for nonlinear and non-stationary time series analysis," Proceedings of the Royal Society of London. Series A: Mathematical, Physical and Engineering Sciences, vol. 454, no. 1971, pp. 903-995, Mar. 1998, doi: 10.1098/rspa.1998.0193.

[15] K. T. Sweeney, S. F. McLoone, and T. E. Ward, "The use of ensemble empirical mode decomposition with canonical correlation analysis as a novel artifact removal technique,” IEEE Transactions on Biomedical Engineering, vol. 60, no. 1, pp. 97-105, Jan. 2013, doi: 10.1109/TBME.2012.2225427.

[16] V. Roy, S. Shukla, P. K. Shukla, and P. Rawat, "Gaussian elimination-based novel canonical correlation analysis method for EEG motion artifact removal," Journal of Healthcare Engineering, vol. 2017, pp. 1-11, 2017, doi: 10.1155/2017/9674712.

[17] P. Gajbhiye, R. K. Tripathy, A. Bhattacharyya, and R. B. Pachori, "Novel approaches for the removal of motion artifact from EEG recordings," IEEE Sensors Journal, vol. 19, no. 22, pp. 10600-10608, Nov. 2019, doi: 10.1109/JSEN.2019.2931727.

[18] A. Bertrand, V. Mihajlovic, B. Grundlehner, C. Van Hoof, and M. Moonen, "Motion artifact reduction in EEG recordings using multi-channel contact impedance measurements," in 2013 IEEE Biomedical Circuits and Systems Conference (BioCAS), Oct. 2013, pp. 258-261, doi: 10.1109/BioCAS.2013.6679688.

[19] V. Mihajlovic, S. Patki, and B. Grundlehner, "The impact of head movements on EEG and contact impedance: An adaptive filtering solution for motion artifact reduction," in 2014 36th Annual International Conference of the IEEE Engineering in Medicine and Biology Society, Aug. 2014, pp. 5064-5067, doi: 10.1109/EMBC.2014.6944763.

[20] K. T. Sweeney, H. Ayaz, T. E. Ward, M. Izzetoglu, S. F. McLoone, and B. Onaral, "A methodology for validating artifact removal techniques for physiological signals," IEEE Transactions on Information Technology in Biomedicine, vol. 16, no. 5, pp. 918-926, Sep. 2012, doi: 10.1109/TITB.2012.2207400

[21] A. Shoeb, "Application of machine learning to epileptic seizure onset detection and treatment," Diss. Massachusetts Inst. Technol, pp. 157-162, 2009.

[22] N. Richer, R. J. Downey, A. D. Nordin, W. D. Hairston, and D. P. Ferris, "Adding neck muscle activity to a head phantom device to validate mobile EEG muscle and motion artifact removal*," in 2019 9th International IEEE/EMBS Conference on Neural Engineering (NER), Mar. 2019, pp. 275-278, doi: 10.1109/NER.2019.8716959.

[23] E. Arad, R. P. Bartsch, J. W. Kantelhardt, and M. Plotnik, "Performance-based approach for movement artifact removal from electroencephalographic data recorded during locomotion," Plos One, vol. 13, no. 5, p. e0197153, May 2018, doi: 10.1371/journal.pone.0197153. 
[24] B. H. Kim and S. Jo, "Real-time motion artifact detection and removal for ambulatory BCI," in The 3rd International Winter Conference on Brain-Computer Interface, Jan. 2015, pp. 1-4, doi: 10.1109/IWW-BCI.2015.7073050.

[25] M. S. Islam, A. M. El-Hajj, H. Alawieh, Z. Dawy, N. Abbas, and J. El-Imad, "EEG mobility artifact removal for ambulatory epileptic seizure prediction applications," Biomedical Signal Processing and Control, vol. 55, p. 101638, Jan. 2020, doi: 10.1016/j.bspc.2019.101638.

[26] B. H. Kim, J. Chun, and S. Jo, "Dynamic motion artifact removal using inertial sensors for mobile BCI," in 2015 7th International IEEE/EMBS Conference on Neural Engineering (NER), Apr. 2015, pp. 37-40, doi: 10.1109/NER.2015.7146554.

[27] S. O'Regan and W. Marnane, "Multimodal detection of head-movement artefacts in EEG," Journal of Neuroscience Methods, vol. 218, no. 1, pp. 110-120, Aug. 2013, doi: 10.1016/j.jneumeth.2013.04.017.

[28] K. Onikura, Y. Katayama, and K. Iramina, "Evaluation of a method of removing head movement artifact from EEG by independent component analysis and filtering," Advanced Biomedical Engineering, vol. 4, pp. 67-72, 2015, doi: $10.14326 \% 2$ Fabe.4.67.

[29] E. Butkeviciute et al., "Removal of movement artefact for mobile EEG analysis in sports exercises," IEEE Access, vol. 7, pp. 7206-7217, 2019, doi: 10.1109/ACCESS.2018.2890335.

[30] V. Mihajlovic and B. Grundlehner, "The effect of force and electrode material on electrode-to-skin impedance," in 2012 IEEE Biomedical Circuits and Systems Conference (BioCAS), Nov. 2012, pp. 57-60, doi: 10.1109/BioCAS.2012.6418511.

\section{BIOGRAPHIES OF AUTHORS}

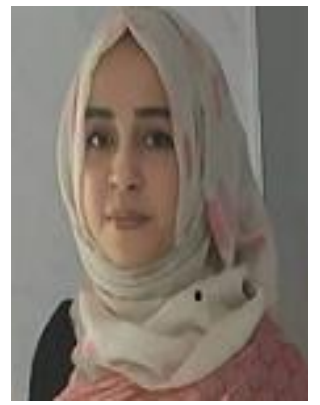

Asma Islam (D) 81 SC P completed her B.Sc. in Electrical and Electronic Engineering from Independent University, Bangladesh in the year 2020. She wants to engage in neuro-signal studies, technological solutions towards mental health and understand brain dynamics. At present, she is continuing her research on how to identify and remove motion artifacts from ambulatory Electroencephalography data. She can be contacted at email: asmaafiyat@gmail.com.

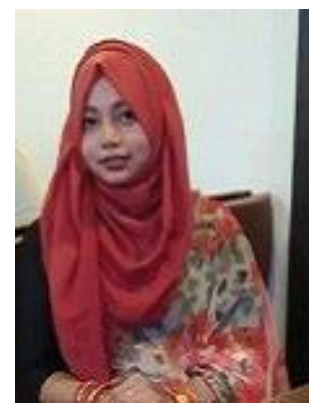

Eshrat Jahan Esha (iD SC P completed her B.Sc. in Electrical and Electronic Engineering from Independent University, Bangladesh in the year 2020. Her research interests include EEG signal processing, biomedical signal processing and neural signal processing. Currently, she is doing research on mobile e-health, especially handling movement artifacts in the wearable scalp EEG recording techniques. She can be contacted at email: eshrat.jahan129@gmail.com.

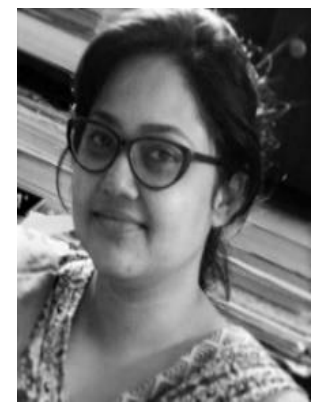

Sheikh Farhana Binte Ahmed (iD IS SC P completed her B.Sc. and M.Sc. in Electrical \& Electronic Engineering from American International University Bangladesh (AIUB) and Islamic University of Technology (IUT) in the year 2012 and 2016 respectively. She is currently pursuing her Ph.D. degree in Electrical and Electronic Engineering from Islamic University of Technology (IUT), Dhaka, Bangladesh. Her research interests include biomedical signal processing, machine learning and control systems. She can be contacted at email: skfarhana.ahmed@gmail.com.

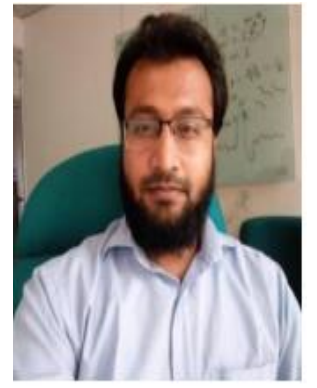

Md. Kafiul Islam (D) RI SC P has received his B.Sc. in EEE from Islamic University of Technology (IUT), Bangladesh in 2008 and completed his PhD from Dept. of ECE, NUS, Singapore in the area of Neural Signal Processing back in 2015. Currently he is serving as an Associate Professor and Course Coordinator in the Dept. of Electrical and Electronic Engineering of Independent University, Bangladesh. His research interests include biomedical instrumentation and signal processing, neural signal processing, brain-computer interface (BCI), etc. He is a Senior Member of IEEE. He has published more than 45 (in total) peer reviewed journal articles, conference papers and book chapters with Google Scholar Citations of 765+ and impact factor contribution of around 20+. Dr. Islam has won several best paper/presentation awards in IEEE conferences. He can be contacted at email: kafiul_islam@iub.edu.bd. 